\title{
A comparison between amblyopic and fellow eyes in unilateral amblyopia using spectral-domain optical coherence tomography
}

This article was published in the following Dove Press journal:

Clinical Ophthalmology

7 November 2014

Number of times this article has been viewed

\author{
Syunsuke Araki' \\ Atsushi Miki ${ }^{1,2}$ \\ Tsutomu Yamashita ${ }^{1,2}$ \\ Katsutoshi Gotol,2 \\ Kazuko Haruishi' \\ Yoshiaki leki' \\ Junichi Kiryu'
}

'Department of Ophthalmology, Kawasaki Medical School, Kurashiki, Japan; ${ }^{2}$ Department of Sensory Science, Faculty of Health Science and Technology, Kawasaki University of Medical Welfare, Kurashiki, Japan
Correspondence: Atsushi Miki

Department of Ophthalmology, Kawasaki Medical School, 577 Matsushima,

Kurashiki, Okayama 70I-0192, Japan

Tel +8186462 IIII

Fax +8I 86464 I565

Email amiki@tc5.so-net.ne.jp
Purpose: To compare the macular retinal thickness and characteristics of optic nerve head $(\mathrm{ONH})$ parameters in amblyopic and fellow eyes in patients with unilateral amblyopia.

Patients and methods: A total of 21 patients with unilateral amblyopia (14 patients with anisometropic amblyopia, four patients with strabismic amblyopia, and three patients with both) were examined using spectral-domain optical coherence tomography. The mean age of the patients was $8.5 \pm 3.5$ years. The examined parameters included the mean macular (full, inner, and outer), ganglion cell complex and circumpapillary retinal nerve fiber layer (cpRNFL) thicknesses, and $\mathrm{ONH}$ parameters (rim volume, nerve head volume, cup volume, rim area, optic disc area, cup area, and cup-to-disc area ratio).

Results: The amblyopic eyes were significantly more hyperopic than the fellow eyes $(P<0.001)$. Among the macular retinal thickness parameters, the cpRNFL thickness $(P<0.01)$, macular full retinal thickness ( $3 \mathrm{~mm}$ region) $(P<0.01)$, and macular outer retinal thickness ( 1 and $3 \mathrm{~mm}$ regions) $(P<0.05)$ were significantly thicker in the amblyopic eyes than in the fellow eyes, while the ganglion cell complex thickness, macular full retinal thickness ( $1 \mathrm{~mm}$ region), and macular inner retinal thickness ( 1 and $3 \mathrm{~mm}$ regions) were not significantly different. Among the $\mathrm{ONH}$ parameters, the rim area was significantly larger and the cup-to-disc area ratio was smaller in the amblyopic eyes than in the fellow eyes $(P<0.05)$. None of the other ONH parameters were significantly different between the investigated eyes. The differences in the cpRNFL thickness and macular outer retinal thickness in the $1 \mathrm{~mm}$ region were significantly correlated with the difference in axial length $(P<0.05, r=-0.48 ; P<0.01, r=-0.59$, respectively) and refractive error $(P<0.05, r=0.50 ; P<0.01, r=0.60$, respectively). The other parameters were not significantly related to the difference in axial length, refractive error, or best corrected visual acuity.

Conclusion: We found significant differences in some of the morphological measurements between amblyopic and fellow eyes that appear to be independent of abnormalities in the visual cortex.

Keywords: anisometropic amblyopia, strabismic amblyopia, ganglion cell complex thickness, macular retinal thickness, peripapillary retinal nerve fiber layer thickness

\section{Introduction}

Amblyopia is a visual disorder characterized by a subnormal visual acuity (VA) and contrast sensitivity in one or both eyes, caused by either visual deprivation or abnormal binocular interactions. ${ }^{1}$ Organic and functional changes in the visual cortex and lateral geniculate nucleus are observed in patients with amblyopia. ${ }^{2-6}$

Although past studies have documented retinal abnormalities in amblyopia patients, ${ }^{7,8}$ the majority of subsequent reports have rejected this opinion. ${ }^{9,10}$ However, in recent years, reinvestigations using fundus photographs have suggested the presence of organic changes in amblyopic eyes, which had previously been assumed to 
be normal. ${ }^{11,12}$ Therefore, whether the retina is normal in amblyopia patients remains to be investigated.

The development of time-domain optical coherence tomography (OCT) (TD-OCT), which analyzes the retinal laminar structure, revealed indispensable information regarding the retinal pathology of macular disease and glaucoma. In addition, in the field of amblyopia, several groups have evaluated the retinal thickness using TD-OCT. ${ }^{13-26}$ For example, Yen et $\mathrm{al}^{13}$ hypothesized that amblyopia may affect the postnatal maturation of the retina, including the postnatal reduction of retinal ganglion cells, which would lead to a measurable increase in the retinal nerve fiber layer (RNFL) thickness in amblyopic eyes. However, the majority of researchers have reported no differences in the RNFL thickness in patients with amblyopia. ${ }^{14,16-26}$

In recent years, the use of spectral-domain OCT (SDOCT), in which the scan speed, spatial resolution, and segmentation technology are improved, has become mainstream, and the stratification of the retinal layer has since become possible, permitting a more detailed assessment of pathologic changes of the retina. Recently, several examinations of the amblyopic retinal thickness using SD-OCT have been reported. ${ }^{27-34}$ In some of these reports, there were no differences in the retinal thickness between amblyopic and fellow eyes, whereas, in others, the amblyopic eyes were found to be either thicker or thinner than the fellow eyes. Therefore, a consensus has not yet been obtained based on OCT, and the degree of retinal involvement accompanying amblyopia is controversial.

In this report, we compared the macula, ganglion cell complex (GCC), and circumpapillary RNFL (cpRNFL) thicknesses, and optic nerve head $(\mathrm{ONH})$ parameters (rim volume, nerve head volume, cup volume, rim area, optic disc area, cup area, and cup-to-disc [C/D] area ratio) between amblyopic and fellow eyes in patients with unilateral amblyopia using SD-OCT.

\section{Material and methods \\ Subjects}

This study was conducted at the Department of Ophthalmology at Kawasaki Medical School Hospital. All of the enrolled patients were diagnosed with unilateral amblyopia and underwent SD-OCT examination. Unilateral amblyopia was defined as a best corrected VA (BCVA) of at least a two-line difference between the amblyopic and fellow eye. The clinical examinations included VA testing, cycloplegic refraction, axial length, slit-lamp examinations, cover and cover-uncover test, extraocular movements, and fundoscopy. The BCVA was transformed to logarithm of the minimum angle of resolution (logMAR) units for the statistical analysis. The axial length was measured using the IOL master ${ }^{\circledR}$ (Carl Zeiss Meditec AG, Jena, Germany). Patients with neurological diseases, ocular conditions such as glaucoma or retinal disorders, and nystagmus were excluded from the study. We adopted SD-OCT data with more than 50 signal strength index and excluded the ones when there was poor fixation or a segmentation error. The cause of amblyopia was anisometropia, strabismus, or both. Anisometropia was defined as an interocular difference in refraction (spherical equivalent) of more than 2.0 diopters (D). The patients with strabismic amblyopia had esotropia or exotropia of at least 10 prism $D$ on the alternate cover test and a spherical difference between the two eyes of less than 1.0 D. A total of 21 patients (ten males and eleven females) with unilateral amblyopia (14 patients with anisometropic amblyopia, four patients with strabismic amblyopia, and three patients with both) were enrolled. The mean age \pm standard deviation (SD) of the patients was $8.5 \pm 3.5$ years (range: $4-18$ years). The mean BCVA was $0.20 \pm 0.30$ $\log$ MAR in the amblyopic eyes and $-0.14 \pm 0.07 \log$ MAR in the fellow eyes. The mean spherical equivalent was $+3.51 \pm 3.60$ $\mathrm{D}$ in the amblyopic eyes and $+0.77 \pm 1.58 \mathrm{D}$ in the fellow eyes. The axial length was $21.94 \pm 1.39 \mathrm{~mm}$ in the amblyopic eyes and $22.99 \pm 0.91 \mathrm{~mm}$ in the fellow eyes (Table 1).

This study adhered to the tenets of the Declaration of Helsinki and was approved by the institutional review board committee of Kawasaki Medical School. Informed consent for the examinations was obtained from each patient or one of each patient's parents.

\section{SD-OCT examinations}

The ONH parameters and thickness of the macula, GCC, and cpRNFL were measured using SD-OCT. The OCT images were obtained using the RTVue- $100^{\circledR}$ (Optovue Inc., Fremont, CA, USA), which acquires 26,000 A-scans per second and provides a $5.0 \mu \mathrm{m}$ depth resolution in the tissue. The RTVue-100 ${ }^{\circledR}$ software package, version 4.0, was used for data acquisition. Adjustment for the refractive error is performed automatically in this device. In patients with poor fixation, the OCT device was operated manually so that the center of fovea or optic disc was located appropriately in each image.

The macular thickness was determined using the MM5 protocol in which data are acquired along a $5 \times 5 \mathrm{~mm}^{2}$ grid of eleven horizontal and eleven vertical lines (each 668 A-scans) at $0.5 \mathrm{~mm}$ intervals and an inner $3 \times 3 \mathrm{~mm}^{2}$ grid of six horizontal and six vertical lines (each $400 \mathrm{~A}$-scans) at $0.5 \mathrm{~mm}$ intervals (Figure 1A). The built-in software program allows for the automated segmentation of the macular full retinal thickness (from the inner limiting membrane [ILM] to the outer border 
Table I Clinical characteristics of the 21 patients with amblyopia included in the study

\begin{tabular}{lllll}
\hline & Amblyopic eyes $(\mathbf{n}=\mathbf{2}$ I) & Fellow eyes $(\mathbf{n}=\mathbf{2} \mathrm{I})$ & $\boldsymbol{P}$-value & $\boldsymbol{t}$-value \\
\hline Refractive error (diopter) & $3.5 \mathrm{I} \pm 3.6$ & $0.77 \pm 1.58$ & $<0.00 \mathrm{I}$ & $4.85 \mathrm{I}$ \\
BCVA (logMAR) & $0.20 \pm 0.30$ & $-0.14 \pm 0.07$ & $<0.00 \mathrm{I}$ & 5.057 \\
Axial length (mm) & $21.94 \pm 1.39$ & $22.99 \pm 0.9 \mathrm{I}$ & $<0.00 \mathrm{I}$ & -4.810 \\
\hline
\end{tabular}

Notes: Values are shown as mean \pm standard deviation. Refractive error: spherical equivalent values. $P$ - and $t$-values are for comparison between amblyopic and fellow eyes (paired $t$-test).

Abbreviations: BCVA, best corrected visual acuity; logMAR, logarithm of the minimal angle of resolution.

of the retinal pigment epithelium [RPE]) as well as the macular inner retinal thickness (from the ILM to the outer boundary of the inner plexiform layer) and macular outer retinal thickness (from the inner border of the inner nuclear layer to the outer border of the RPE) (Figure 1B). The retinal macular thickness was measured in two regions, which included regions within $1 \mathrm{~mm}$ ("1 mm" region) and between 1 and $3 \mathrm{~mm}$ ("3 mm" region) from the center (Figure 1C).

The GCC protocol was used to obtain the macular measurements. This protocol consists of one horizontal line scan measuring $7 \mathrm{~mm}$ in length (467 A-scans) and 15 vertical line scans measuring $7 \mathrm{~mm}$ in length (each $400 \mathrm{~A}$-scans) obtained at $0.5 \mathrm{~mm}$ intervals (Figure 2A). The GCC is measured from the ILM to the outer boundary of the inner plexiform layer (IPL), similar to the macular inner retinal thickness in MM5. The center of the GCC scan is shifted $0.75 \mathrm{~mm}$ temporally to improve sampling of the temporal periphery as part of the glaucoma-analysis program. This scan configuration provides 14,810 A-scans in 0.58 seconds.

The ONH parameters and cpRNFL thickness values were determined using the ONH map $4 \mathrm{~mm}$ protocol in the threedimensional baseline mode, in which data acquired along a $3.45 \mathrm{~mm}$ diameter circle around the optic disc are recalculated with a map created from en face imaging that used six circular scans ranging from a 2.5 to $4.0 \mathrm{~mm}$ region ( 587 or $775 \mathrm{~A}$-scans each) centered on the optic disc and 12 linear radial scan data inputs (3.4 mm in length, $452 \mathrm{~A}$-scans each) (Figure 2B). This scan protocol provides $9,510 \mathrm{~A}$-scans in 0.39 seconds.

\section{Statistical analysis}

The paired $t$-test was used to determine whether the differences between the values of the amblyopic and fellow eyes were significant. $P$-values of less than 0.05 were considered to be statistically significant.

\section{A}
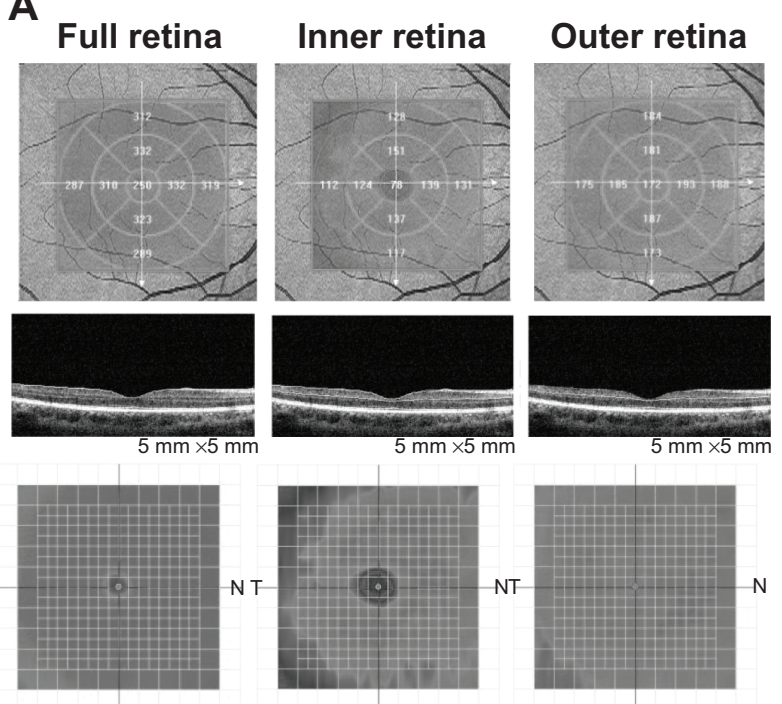

Figure I Measurement of the MM5 in a normal subject using RTVue- $100^{\circledR}$.

Notes: (A) MM5 protocol. (B) ILM-IPL: from the ILM to the outer boundary of the IPL. INL-RPE: from the inner border of the INL to the outer border of the RPE. ILMRPE: from the ILM to the outer border of the RPE. (C) "I mm" is a region of within I mm of the diameter from the center. " $3 \mathrm{~mm}$ " is a region of between I and $3 \mathrm{~mm}$ of the diameter from the center.

Abbreviations: ILM, inner limiting membrane; IPL, inner plexiform layer; INL, inner nuclear layer; RPE, retinal pigment epithelium; S, superior; N, nasal; I, inferior; T, temporal. 
A

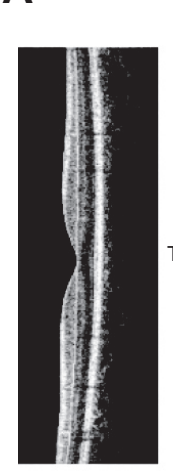

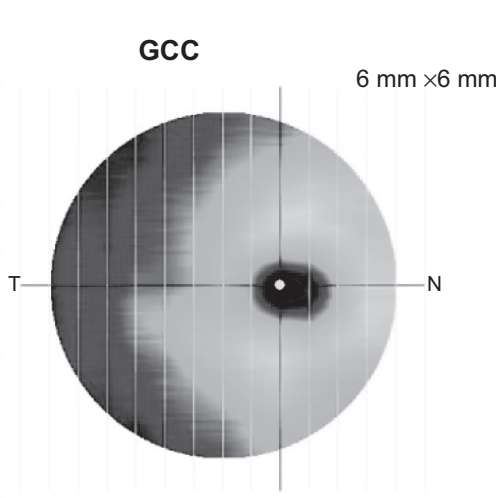

B

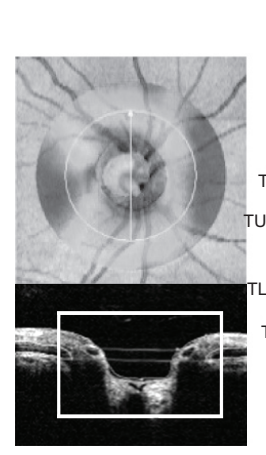

ONH

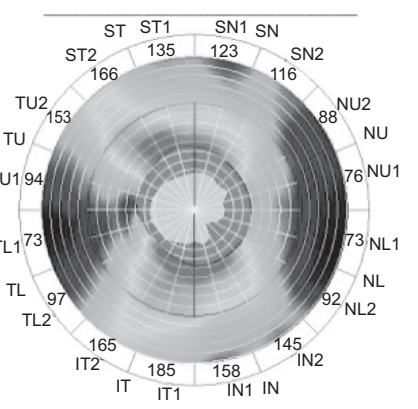

Figure 2 Measurement of the GCC and ONH in a normal subject using RTVue- $100^{\circledR}$.

Notes: (A) GCC protocol. (B) ONH protocol. The ONH measurements are performed within the area marked with the line.

Abbreviations: GCC, ganglion cell complex; ONH, optic nerve head; T, temporal; N, nasal; SN, superior nasal; NU, nasal upper; NL, nasal lower; IN, inferior nasal; IT, inferior temporal; TL, temporal lower; TU, temporal upper; ST, superior temporal.

The correlation between the differences in the axial length, refractive error, and BCVA versus the differences in the SD-OCT parameters were analyzed using the Spearman's rank correlation coefficient. $P$-values of less than 0.05 were considered to be statistically significant.

There is a possibility that a difference of refraction and the axis length might have affected the difference of the retinal thickness. Therefore, we examined whether the retinal thickness, which showed a difference between amblyopic eyes and fellow eyes, was associated with axial length, refractive error, or BCVA.

\section{Results}

Table 2 shows the mean $( \pm \mathrm{SD})$ values of the GCC and cpRNFL thicknesses and the ONH parameters (rim volume, nerve head volume, cup volume, rim area, optic disc area, cup area, average $\mathrm{C} / \mathrm{D}$ ratio, vertical $\mathrm{C} / \mathrm{D}$ ratio, and horizontal C/D ratio) obtained on SD-OCT. The mean cpRNFL thickness was significantly thicker in the amblyopic eyes than in the fellow eyes $(P<0.01)$, while the GCC thickness was not significantly different between the two eyes. Among the ONH parameters, the rim area was significantly larger $(P<0.05)$, and the $\mathrm{C} / \mathrm{D}$ ratio was smaller, in the amblyopic eyes than in the fellow eyes $(P<0.05)$. None of the other ONH parameters were significantly different.

Table 3 shows the mean $( \pm \mathrm{SD})$ values of the macular full, inner, and outer retinal thickness (1 and $3 \mathrm{~mm}$ regions) obtained on SD-OCT. The mean macular full retinal thickness ( $3 \mathrm{~mm}$ region) and macular outer retinal thickness (1 and 3 mm regions) were significantly thicker in the amblyopic eyes than the fellow eyes $(P<0.01$ and $P<0.05$, respectively), whereas the macular full retinal thickness (1 $\mathrm{mm}$ region) and macular inner retinal thickness ( 1 and $3 \mathrm{~mm}$ regions) were not significantly different.

Figure 3 shows the correlations between the differences in axial length, refractive error, and BCVA versus the

Table 2 Comparison of the cpRNFL and GCC thickness values and ONH parameters between the amblyopic and fellow eyes

\begin{tabular}{|c|c|c|c|c|}
\hline & Amblyopic eyes $(n=21)$ & Fellow eyes $(n=2 I)$ & $P$-value & $t$-value \\
\hline cpRNFL thickness $(\mu \mathrm{m})$ & $112.5 \pm 9.21$ & $107.03 \pm 8.74$ & 0.004 & 3.252 \\
\hline GCC thickness $(\mu \mathrm{m})$ & $96.8 \pm 7.02$ & $95.72 \pm 7.01$ & 0.107 & 1.686 \\
\hline \multicolumn{5}{|l|}{ ONH parameters } \\
\hline Rim volume $\left(\mathrm{mm}^{3}\right)$ & $0.199 \pm 0.084$ & $0.195 \pm 0.131$ & 0.862 & 0.176 \\
\hline Nerve head volume $\left(\mathrm{mm}^{3}\right)$ & $0.338 \pm 0.125$ & $0.328 \pm 0.192$ & 0.717 & 0.368 \\
\hline Cup volume $\left(\mathrm{mm}^{3}\right)$ & $0.167 \pm 0.174$ & $0.191 \pm 0.193$ & 0.372 & -0.914 \\
\hline $\operatorname{Rim}$ area $\left(\mathrm{mm}^{2}\right)$ & $1.53 \pm 0.27$ & $1.35 \pm 0.42$ & 0.037 & 2.240 \\
\hline Optic disc area $\left(\mathrm{mm}^{2}\right)$ & $2.26 \pm 0.46$ & $2.23 \pm 0.45$ & 0.752 & 0.320 \\
\hline Cup area $\left(\mathrm{mm}^{2}\right)$ & $0.73 \pm 0.55$ & $0.88 \pm 0.63$ & 0.120 & -1.624 \\
\hline Average $C / D$ ratio & $0.29 \pm 0.17$ & $0.37 \pm 0.22$ & 0.024 & -2.442 \\
\hline Vertical C/D ratio & $0.5 I \pm 0.16$ & $0.58 \pm 0.19$ & 0.016 & -2.626 \\
\hline Horizontal C/D ratio & $0.60 \pm 0.19$ & $0.67 \pm 0.23$ & 0.015 & -2.669 \\
\hline
\end{tabular}

Notes: Values are shown as mean \pm standard deviation. $P$ - and $t$-values are for comparison between amblyopic and fellow eyes (paired $t$-test). Abbreviations: $C / D$, cup-to-disc; cpRNFL, circumpapillary retinal nerve fiber layer; GCC, ganglion cell complex; $\mathrm{ONH}$, optic nerve head. 
Table 3 Comparison of the macular retinal thickness between the amblyopic and fellow eyes

\begin{tabular}{|c|c|c|c|c|}
\hline & Amblyopic eyes $(n=2 I)$ & Fellow eyes $(n=2 I)$ & $P$-value & t-value \\
\hline \multicolumn{5}{|c|}{ Macular full retinal thickness $(\mu \mathrm{m})$} \\
\hline $\mathrm{I} \mathrm{mm}$ & $236.90 \pm 18.11$ & $231.67 \pm 15.17$ & 0.099 & 1.730 \\
\hline $3 \mathrm{~mm}$ & $313.33 \pm 14.97$ & $310.14 \pm 12.68$ & 0.009 & 2.915 \\
\hline \multicolumn{5}{|c|}{ Macular inner retinal thickness $(\mu \mathrm{m})$} \\
\hline $\mathrm{I} \mathrm{mm}$ & $69.57 \pm 9.97$ & $67.81 \pm 9.44$ & 0.379 & 0.900 \\
\hline $3 \mathrm{~mm}$ & $131.52 \pm 9.01$ & $|30.7| \pm 6.32$ & 0.545 & 0.615 \\
\hline \multicolumn{5}{|c|}{ Macular outer retinal thickness $(\mu \mathrm{m})$} \\
\hline $\mathrm{I} \mathrm{mm}$ & $167.33 \pm 10.89$ & $163.81 \pm 8.16$ & 0.034 & 2.278 \\
\hline $3 \mathrm{~mm}$ & $181.95 \pm 10.52$ & $179.52 \pm 8.49$ & 0.038 & 2.219 \\
\hline
\end{tabular}

Notes: Values are shown as mean \pm standard deviation. $P$ - and $t$-values are for comparison between amblyopic and fellow eyes (paired $t$-test). "I mm” is a region within I mm of the diameter from the center. " $3 \mathrm{~mm}$ " is a region of between I and $3 \mathrm{~mm}$ of the diameter from the center.

differences in the cpRNFL, macular retinal thicknesses, and rim area. The differences in the cpRNFL thickness and macular outer retinal thickness in the $1 \mathrm{~mm}$ region were significantly correlated with the difference in axial length $(P<0.05, r=-0.48 ; P<0.01, r=-0.59$, respectively) and refractive error $(P<0.05, r=0.50 ; P<0.01, r=0.60$, respectively). The other parameters were not significantly related to the difference in axial length, refractive error, or BCVA.

\section{Discussion}

In this study, we compared the retinal thicknesses and $\mathrm{ONH}$ parameters in patients with unilateral amblyopia between the amblyopic eye and fellow eye using SD-OCT. The cause of amblyopia was anisometropia (67\%), strabismus (19\%), or both (14\%). Consequently, we found significant differences in some of the parameters; such differences were most prominent in the cpRNFL and macular full retinal thickness ( $3 \mathrm{~mm}$ region), in which these values were significantly thicker in the amblyopic eyes than in the fellow eyes. In addition, we found that the macular outer retinal thickness was thicker in the amblyopic eyes than in the fellow eyes.

Furthermore, we found that the cpRNFL thickness was significantly thicker in the amblyopic eyes than in the fellow eyes. In previous studies, Yen et $\mathrm{al}^{13}$ hypothesized that amblyopia affects the postnatal maturation of the retina, including the postnatal reduction of retinal ganglion cells, which would lead to a measurable increase in the RNFL thickness in amblyopic eyes measured using TD-OCT. In addition, $\mathrm{Wu}$ et $\mathrm{a}^{31}$ reported that anisometropic amblyopes had thicker cpRNFL values in the amblyopic eye than in the fellow eye based on SD-OCT. Our present data is in agreement with these results. In contrast, some studies that have investigated the cpRNFL thickness in amblyopic eyes using SD-OCT found no significant differences compared to that observed in controls..$^{27,29,32}$ Regarding this contradiction, Kim et $\mathrm{al}^{34}$ pointed out that the majority of these studies did not correct the ocular magnification errors related to refraction and axial length, whereas most previous studies included patients with anisometropia. In the present study, we included such patients, and the difference in cpRNFL thickness between the two eyes was found to be significantly related to the difference in axial length and refractive error. Tsai et a ${ }^{35}$ demonstrated that the spherical equivalent refraction is a significant predictor of the pediatric RNFL thickness. However, Wang et $\mathrm{a}^{36}$ reported that the effects of magnification attributable to the axial length and refractive error on the measurement of the RNFL thickness were not statistically significant. Therefore, although we cannot exclude the possibility that axial length and refractive error may have affected the cpRNFL thickness in this study, it is unclear to what degree any axial length and refractive differences had an influence on the difference in the cpRNFL thickness. On the other hand, the difference in the cpRNFL thickness between the two eyes was not significantly related to the difference in BCVA. Therefore, there is a possibility that factors other than the visual function have an effect on the cpRNFL thickness.

Regarding the ONH parameters, the rim area was significantly larger and the $\mathrm{C} / \mathrm{D}$ ratio was smaller in the amblyopic eyes than in the fellow eyes. In addition, the difference in the rim area was not correlated with that of refraction or axial length. The changes in these two parameters may be associated with an increase in the RNFL thickness. In addition, it has been reported that the number of optic nerve fibers in animal models of experimental glaucoma shows a high correlation with the $\mathrm{C} / \mathrm{D}$ ratio and rim area of the optic disc..$^{37,38}$ Furthermore, Kanamori et $\mathrm{al}^{39}$ reported that the rim area and cpRNFL thickness exhibit a positive straight-line correlation in glaucomatous eyes. While these reports examined patients with glaucoma, our results demonstrated that a 

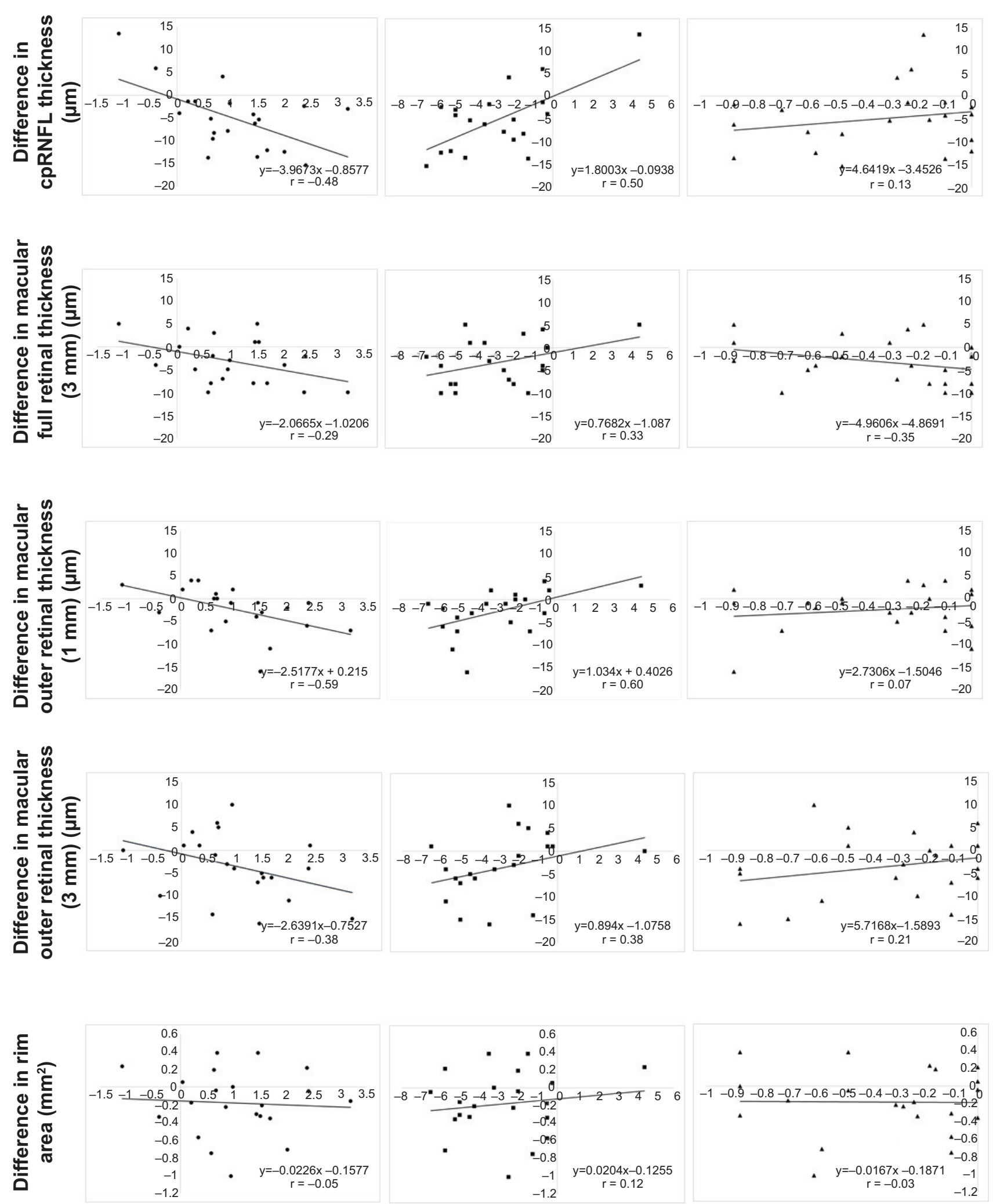

Difference in axial length ( $\mathrm{mm})$ Difference in refractive error (D) Difference in BCVA (logMAR)

Figure 3 Correlations between the differences in the axial length, refractive error, and BCVA versus the differences in the SD-OCT parameters between the amblyopic and fellow eyes.

Notes: The difference value shows deducted measurements of amblyopic eyes from those of fellow eyes. The differences in the cpRNFL thickness and macular outer retinal thickness in the I mm region were significantly correlated with the difference in axial length $(P<0.05, r=-0.48 ; P<0.01, r=-0.59$, respectively) and refractive error $(P<0.05$, $r=0.50 ; P<0.01, r=0.60$, respectively). The other parameters were not significantly related to the difference in axial length, refractive error, or $B C V A$.

Abbreviations: BCVA, best corrected visual acuity; CPRNFL, circumpapillary retinal nerve fiber layer; logMAR, logarithm of the minimal angle of resolution; SD-OCT, spectral domain optical coherence tomography. 
similar correlation can be found in patients with amblyopia. Meanwhile, Kim et a ${ }^{34}$ reported that $\mathrm{ONH}$ parameters show no significant differences between amblyopic and fellow eyes in patients with deprivational amblyopia. Therefore, it is necessary to segregate such patients into groups according to the type of amblyopia in further investigations.

On the other hand, the GCC and macular inner retinal thickness did not display any significant differences between the amblyopic and fellow eyes in this study. The GCC and macular inner retinal thickness consists of the RNFL, ganglion cell layer (GCL), and IPL. In previous studies of the inner retinal thickness using SD-OCT, Firat et al ${ }^{32}$ showed no significant differences in GCC thickness between amblyopic eyes and controls. On the other hand, Park et a ${ }^{28}$ reported that the GCL-IPL thickness was thinner in amblyopic eyes than in fellow eyes. In contrast, Tugcu et $\mathrm{al}^{33}$ found that the GCC thickness was thicker in amblyopic eyes than in controls. The causes of these differences in results may be related to factors in the study design, such as which structures were measured; for example, whether the nerve fiber layer thickness was included in the inner retinal thickness. Additionally, that heterogeneity of the SD-OCT model that was used for the study is one of the factors to cause the different results.

In the present study, the GCC and macular inner retinal thickness values were not significantly different, unlike that observed for the cpRNFL thickness. These results are similar to those obtained by Kim et al. ${ }^{34}$ Furthermore, Yoon et al ${ }^{15}$ reported that the RNFL in patients with amblyopia was significantly thicker, although there were no significant differences in macular thickness, based on TD-OCT. In humans, the total population of cells in the GCL and number of axons in the optic nerve decreases during gestation. ${ }^{40,41}$ If amblyopia affects the process of postnatal reduction of the retina, the amblyopic effects may involve the morphology of the circumpapillary region, including the cpRNFL thickness and rim area. However, this hypothesis does not explain why there were no changes in the macular GCC values.

The macular full retinal thickness in the $3 \mathrm{~mm}$ region was found to be significantly thicker in the amblyopic eyes than in the fellow eyes, whereas that in the $1 \mathrm{~mm}$ region was not significantly different between the two groups. Among previous studies using SD-OCT, most reported that there were no differences in macular thickness between amblyopic and normal fellow eyes, ${ }^{28,29,31-34}$ although some showed that the macular thickness in the amblyopic eyes was greater than that observed in the normal fellow eyes..$^{27,30}$ Wu et al ${ }^{31}$ reported that the central macular thickness values in the $1 \mathrm{~mm}$ and $3 \mathrm{~mm}$ regions were not significantly different in amblyopic and normal fellow eyes, although the mean macular foveolar thickness was significantly thicker in the amblyopic eyes than in the fellow eyes. We did not examine the foveolar thickness in this study; however, there were no differences in the macular full retinal thickness in the $1 \mathrm{~mm}$ region, similar to that observed in most previous studies. ${ }^{28,29,31-34}$ However, the macular full retinal thickness in the $3 \mathrm{~mm}$ region was significantly thicker in the amblyopic eyes than in the fellow eyes. This difference seems to have originated from structural changes in the outer retina, as the inner macular retinal (GCC) thickness values were not different in this study. This result is in disagreement with that of Wu et al in the $3 \mathrm{~mm}$ region. ${ }^{31} \mathrm{In}$ the present report, the difference in the macular full retinal thickness in the $3 \mathrm{~mm}$ region between the two eyes was not significantly related to that of the axial length or refractive error. In addition, when eyes with pathologic myopia were excluded in previous studies, the axial length and refractive error were found to not affect the macular retinal thickness. ${ }^{42,43}$ Therefore, the effect of the difference in the measurement range (ie, $1 \mathrm{~mm}$ or $3 \mathrm{~mm}$ regions) on the results requires further investigation.

In this study, we found that the macular outer retinal thickness ( $1 \mathrm{~mm}$ and $3 \mathrm{~mm}$ region) was significantly thicker in the amblyopic eyes than in the fellow eyes. Park et al ${ }^{28}$ investigated the thickness of each retinal layer, including the outer retina, by measuring the distance between two retinal points set manually in amblyopic and fellow eyes. However, to the best of our knowledge, the mapping of the outer retinal thickness using the automatic segmentation method has not yet been previously employed. In the present analysis, the difference in the $1 \mathrm{~mm}$-region thickness between contralateral eyes was significantly related to the difference in axial length and refractive error. However, the difference in the $3 \mathrm{~mm}$ region thickness was not significantly related to the difference in axial length or refractive error. We cannot exclude the possibility that the relatively small number of subjects studied resulted in the inability to show a statistically significant relationship. However, although there is a possibility that amblyopia affects the outer retina, including photoreceptor cells, it is unlikely that the visual cortex influences the outer retina without affecting the inner retina.

Our study is associated with some limitations. First, the relatively small number of subjects studied did not allow for segregation of the patients into groups according to the type of amblyopia, refractive error, or age. Second, we did not divide the patients into those with persistent and recovered amblyopia. Third, we compared the amblyopic eye with the fellow eye in the same patient with unilateral amblyopia; however, there was no normal group for comparison. Wang et $\mathrm{al}^{44}$ stated that the fellow eye may not be normal on OCT 
in patients with anisometropic amblyopia. Furthermore, most of the differences in retinal thickness were less than $5 \mu \mathrm{m}$ which is equivalent to the spatial resolution of the instrument. Accordingly, it is unclear if these results are real or a consequence of the limits of the instrumentation. Further OCT studies with higher spatial resolution will resolve this issue.

\section{Conclusion}

In conclusion, we found significant differences in some of the morphological measurements between the amblyopic and fellow eyes in patients with unilateral amblyopia. The cpRNFL thickness and rim area were greater in the amblyopic eyes than in the fellow eyes, whereas the GCC and macular inner retinal thickness values showed no significant differences. Furthermore, the macular full retinal thickness in the $3 \mathrm{~mm}$ region and outer retinal thickness in the $1 \mathrm{~mm}$ and $3 \mathrm{~mm}$ regions were significantly thicker in the amblyopic eyes than in the fellow eyes. However, these differences between amblyopic and fellow eyes appear to be independent of abnormalities in the visual cortex.

\section{Disclosure}

The authors report no conflicts of interest in this work.

\section{References}

1. von Noorden GK. Amblyopia: a multidisciplinary approach. Proctor lecture. Invest Ophthalmol Vis Sci. 1985;26(12):1704-1716.

2. Wiesel TN, Hubel DH. Single-cell responses in striate cortex of kittens deprived of vision in one eye. J Neurophysiol. 1963;26:1003-1017.

3. von Noorden GK. Histological studies of the visual system in monkeys with experimental amblyopia. Invest Ophthalmol. 1973; 12(10):727-738.

4. von Noorden GK, Middleditch PR. Histology of the monkey lateral geniculate nucleus after unilateral lid closure and experimental strabismus: further observations. Invest Ophthalmol. 1975;14(9): 674-683.

5. Miki A, Liu GT, Goldsmith ZG, Liu CS, Haselgrove JC. Decreased activation of the lateral geniculate nucleus in a patient with anisometropic amblyopia demonstrated by functional magnetic resonance imaging. Ophthalmologica. 2003;217(5):365-369.

6. Hess RF, Thompson B, Gole G, Mullen KT. Deficient responses from the lateral geniculate nucleus in humans with amblyopia. Eur JNeurosci. 2009;29(5):1064-1070.

7. McNeil NL. Patterns of visual defects in children. Br J Ophthalmol. 1955;39(11):688-701.

8. Ikeda H, Tremain KE. Amblyopia occurs in retinal ganglion cells in cats reared with convergent squint without alternating fixation. Exp Brain Res. 1979;35(3):559-582.

9. von Noorden GK, Crawford ML, Middleditch PR. Effect of lid suture on retinal ganglion cells in Macaca mulatta. Brain Res. 1977; 122(3):437-444.

10. Hess RF. Amblyopia: site unseen. Clin Exp Optom. 2001;84(6): 321-336.

11. Lempert P. Optic nerve hypoplasia and small eyes in presumed amblyopia. J AAPOS. 2000;4(5):258-266.
12. Lempert $P$. The axial length/disc area ratio in anisometropic hyperopic amblyopia: a hypothesis for decreased unilateral vision associated with hyperopic anisometropia. Ophthalmology. 2004;111(2):304-308.

13. Yen MY, Cheng CY, Wang AG. Retinal nerve fiber layer thickness in unilateral amblyopia. Invest Ophthalmol Vis Sci. 2004;45(7):2224-2230.

14. Altinas O, Yüksel N, Ozkan B, Caglar Y. Thickness of the retinal nerve fiber layer, macular thickness, and macular volume in patients with strabismic amblyopia. J Pediatr Ophthalmol Strabismus. 2005; 42(4):216-221.

15. Yoon SW, Park WH, Baek SH, Kong SM. Thicknesses of macular retinal layer and peripapillary retinal nerve fiber layer in patients with hyperopic anisometropic amblyopia. Korean J Ophthalmol. 2005;19(1):62-67.

16. Repka MX, Goldenberg-Cohen N, Edwards AR. Retinal nerve fiber layer thickness in amblyopic eyes. Am J Ophthalmol. 2006; 142(2):247-251.

17. Kee SY, Lee SY, Lee YC. Thicknesses of the fovea and retinal nerve fiber layer in amblyopic and normal eyes in children. Korean J Ophthalmol. 2006;20(3):177-181.

18. Huynh SC, Samarawickrama C, Wang XY, et al. Macular and nerve fiber layer thickness in amblyopia: the Sydney Childhood Eye Study. Ophthalmology. 2009;116(9):1604-1609.

19. Quoc EB, Delepine B, Tran TH. [Thickness of retinal fiber layer and macular volume in children and adults with strabismic and anisometropic amblyopia]. J Fr Ophtalmol. 2009;32(7):488-495. French.

20. Dickmann A, Petroni S, Salerni A, Dell'Omo R, Balestrazzi E. Unilateral amblyopia: an optical coherence tomography study. J AAPOS. 2009;13(2):148-150.

21. Repka MX, Kraker RT, Tamkins SM, et al. Retinal nerve fiber layer thickness in amblyopic eyes. Am J Ophthalmol. 2009;148(1):143-147.

22. Miki A, Shirakashi M, Yaoeda K, et al. Retinal nerve fiber layer thickness in recovered and persistent amblyopia. Clin Ophthalmol. 2010; 20(4):1061-1064.

23. Walker RA, Rubab S, Voll AR, Erraguntla V, Murphy PH. Macular and peripapillary retinal nerve fibre layer thickness in adults with amblyopia. Can J Ophthalmol. 2011;46(5):425-427.

24. Ersan I, Zengin N, Bozkurt B, Ozkagnici A. Evaluation of retinal nerve fiber layer thickness in patients with anisometropic and strabismic amblyopia using optical coherence tomography. J Pediatr Ophthalmol Strabismus. 2013;50(2):113-117.

25. Xu J, Lu F, Liu W, Zhang F, Chen W, Chen J. Retinal nerve fibre layer thickness and macular thickness in patients with esotropic amblyopia. Clin Exp Optom. 2013;96(3):267-271.

26. Andalib D, Javadzadeh A, Nabai R, Amizadeh Y. Macular and retinal nerve fiber layer thickness in unilateral anisometropic or strabismic amblyopia. J Pediatr Ophthalmol Strabismus. 2013;50(4): 218-221.

27. Al-Haddad CE, El Mollayess GM, Cherfan CG, Jaafar DF, Bashshur ZF. Retinal nerve fiber layer and macular thickness in amblyopia as measured by spectral-domain optical coherence tomography. Br J Ophthalmol. 2011;95(12):1696-1699.

28. Park KA, Park DY, Oh SY. Analysis of spectral-domain optical coherence tomography measurements in amblyopia: a pilot study. Br J Ophthalmol. 2011;95(12):1700-1706.

29. Dickmann A, Petroni S, Perrotta V, et al. Measurement of retinal nerve fiber layer thickness, macular thickness, and foveal volume in amblyopic eyes using spectral-domain optical coherence tomography. $J$ AAPOS. 2012;16(1):86-88.

30. Al-Haddad CE, El Mollayess GM, Mahfoud ZR, Jaafar DF, Bashshur ZF. Macular ultrastructural features in amblyopia using high-definition optical coherence tomography. $\mathrm{Br} J$ Ophthalmol. 2013;97(3):318-322.

31. Wu SQ, Zhu LW, Xu QB, Xu JL, Zhang Y. Macular and peripapillary retinal nerve fiber layer thickness in children with hyperopic anisometropic amblyopia. Int J Ophthalmol. 2013;6(1):85-89. 
32. Firat PG, Ozsoy E, Demirel S, Cumurcu T, Gunduz A. Evaluation of peripapillary retinal nerve fiber layer, macula and ganglion cell thickness in amblyopia using spectral optical coherence tomography. Int J Ophthalmol. 2013;6(1):90-94.

33. Tugcu B, Araz-Ersan B, Kilic M, Erdogan ET, Yigit U, Karamursel S. The morpho-functional evaluation of retina in amblyopia. Curr Eye Res. 2013;38(7):802-809.

34. Kim YW, Kim SJ, Yu YS. Spectral-domain optical coherence tomography analysis in deprivational amblyopia: a pilot study with unilateral pediatric cataract patients. Graefes Arch Clin Exp Ophthalmol. 2013; 251(12):2811-2819.

35. Tsai DC, Huang N, Hwu JJ, Jueng RN, Chou P. Estimating retinal nerve fiber layer thickness in normal schoolchildren with spectral-domain optical coherence tomography. Jpn J Ophthalmol. 2012;56(4):362-370.

36. Wang XY, Huynh SC, Burlutsky G, Ip J, Stapleton F, Mitchell P. Reproducibility of and effect of magnification on optical coherence tomography measurements in children. Am J Ophthalmol. 2007; 143(3):484-488.

37. Varma R, Quigley HA, Pease ME. Changes in optic disc characteristics and number of nerve fibers in experimental glaucoma. Am JOphthalmol. 1992;114(5):554-559.

38. Yücel YH, Gupta N, Kalichman MW, et al. Relationship of optic disc topography to optic nerve fiber number in glaucoma. Arch Ophthalmol. 1998;116(4):493-497.
39. Kanamori A, Kusuhara A, Tatsumi Y, et al. [Correlations among GDx-variable corneal compension, optical coherence tomography, and Heidelberg retina tomograph and relationships between these structural parameters and visual field indices]. Nihon Ganka Gakkai Zasshi. 2006;110(3):180-187. Japanese.

40. Provis JM, van Driel D, Billson FA, Russell P. Development of the human retina: patterns of cell distribution and redistribution in the ganglion cell layer. J Comp Neurol. 1985;233(4):429-451.

41. Provis JM, van Driel D, Billson FA, Russell P. Human fetal optic nerve: overproduction and elimination of retinal axons during development. J Comp Neurol. 1985;238(1):92-100.

42. Wakitani Y, Sasoh M, Sugimoto M, Ito Y, Ido M, Uji Y. Macular thickness measurements in healthy subjects with different axial lengths using optical coherence tomography. Retina. 2003;23(2):177-182.

43. Tewari HK, Wagh VB, Sony P, Venkatesh P, Singh R. Macular thickness evaluation using the optical coherence tomography in normal Indian eyes. Indian J Ophthalmol. 2004;52(3):199-204.

44. Wang BZ, Taranath D. A comparison between the amblyopic eye and normal fellow eye ocular architecture in children with hyperopic anisometropic amblyopia. J AAPOS. 2012;16(5):428-430.
Clinical Ophthalmology

\section{Publish your work in this journal}

Clinical Ophthalmology is an international, peer-reviewed journa covering all subspecialties within ophthalmology. Key topics include: Optometry; Visual science; Pharmacology and drug therapy in eye diseases; Basic Sciences; Primary and Secondary eye care; Patien Safety and Quality of Care Improvements. This journal is indexed on

Submit your manuscript here: http://www.dovepress.com/clinical-ophthalmology-journal

\section{Dovepress}

PubMed Central and CAS, and is the official journal of The Society of Clinical Ophthalmology (SCO). The manuscript management system is completely online and includes a very quick and fair peer-review system, which is all easy to use. Visit http://www.dovepress.com/ testimonials.php to read real quotes from published authors. 